\title{
New pharmacological and technological management strategies in heart failure
}

\author{
This article was published in the following Dove Press journal: \\ Vascular Health and Risk Management \\ 21 March 2017 \\ Number of times this article has been viewed
}

\author{
Sunit-Preet Chaudhry' \\ Garrick C Stewart ${ }^{2}$ \\ 'Division of Cardiology, St Vincent \\ Indianapolis, Indianapolis, IN, ${ }^{2}$ Division \\ of Cardiovascular Medicine, Center \\ for Advanced Heart Disease, Brigham \\ and Women's Hospital, Boston, \\ MA, USA
}

\begin{abstract}
Heart failure is a complex clinical syndrome resulting from impairment of ventricular filling or ejection of blood associated with symptoms of dyspnea, fatigue, as well as peripheral and/or pulmonary edema. This syndrome is progressive and characterized by worsening quality of life despite escalating levels of care, affecting 5.7 million Americans with an annual cost of over $\$ 30$ billion US dollars. Treatment for this syndrome has evolved over three distinct eras: the nonpharmacological era, the pharmacological era, and the device era, with the focus shifting from symptomatic relief to decreasing morbidity and mortality. Over the past 10 years, the field has undergone a renaissance, with the development of new pharmacologic, hemodynamic monitoring, and device therapies proven to improve outcomes in patients with heart failure. This article will review several recent innovations in the management of patients with heart failure.
\end{abstract}

Keywords: heart failure, heart-assist devices, disease management

\section{Introduction}

Heart failure is a complex clinical syndrome resulting from impairment of ventricular filling or ejection of blood associated with symptoms of dyspnea, fatigue, as well as peripheral and/or pulmonary edema. ${ }^{1}$ This syndrome currently affects 5.7 million Americans and its prevalence is expected to increase by $46 \%$ to over 8 million Americans by the year $2030 .^{2}$ It has become an important area of focus within the field of medicine, responsible for $12-15$ million outpatient office visits per year, ${ }^{3}$ and remains one of the most common reasons for admission in patients over the age of $65 .{ }^{4}$

Over the past 50 years, there have been three distinct eras relating to heart failure. ${ }^{5}$ The earliest era, known as the nonpharmacologic era, focused its treatments on bed rest, inactivity, and fluid restriction. This was followed by the pharmacologic era, with two distinct periods: early, marked by the increased use of inotropes and diuretics and the discovery of vasodilators, and late, with the discovery of medications relating to neurohormonal pathways. The current era is the device era, with the discovery, acceptance, and increased use of implantable cardioverter defibrillators, cardiac resynchronization therapy, and left ventricular assist devices (LVADs).

Despite the evolving understanding of heart failure pathophysiology and major therapeutic innovations during these eras, a diagnosis of heart failure continues to carry with it considerable morbidity and mortality. There is a nearly $25 \%$ readmission rate for patients with the heart failure syndrome within 30 days of hospital discharge coupled with an estimated 5-year mortality of $50 \%{ }^{6}$ collectively leading to direct costs of over 30 billion dollars per year. ${ }^{7}$ Given these significant impacts on health care, there has been growing interest in therapies that may decrease morbidity, mortality, along

Vascular Health and Risk Management 2017:13 III-12I

I I I 
with the substantial health care expenditures associated with the disease. These therapies hope to target both patients with heart failure with preserved ejection fraction and those with heart failure with reduced ejection fraction.

Recently, there has been a paradigm shift in the field of heart failure, with new emerging therapeutic options including pharmacologic therapies, outpatient hemodynamic monitoring, and device therapies, resulting in significant improvements in patient outcomes in pivotal clinical studies. This review article will discuss these new therapeutic discoveries as well as provide insight into future therapies.

\section{Pharmacologic therapies}

Pharmacologic therapy has been the cornerstone of management for the heart failure syndrome. Early treatments, consisting of digoxin and diuretics, were aimed primarily at relieving the congestive symptoms. The role of pharmacologic therapy has evolved from simply providing symptom relief to improving morbidity and mortality ever since the release of the first large-scale randomized trial that showed that hydralazine and isosorbide dinitrate decreased mortality in patients with advanced heart failure. ${ }^{8}$ Since this time, there have been three major classes of medications, angiotensinconverting enzyme (ACE) inhibitors, beta blockers, and mineralocorticoid receptor antagonists, which have been shown to decrease mortality in systolic heart failure by $17 \%$, $34 \%$, and $30 \%$, respectively. ${ }^{9}$ Several innovative pharmacotherapies have recently been approved for the treatment of systolic heart failure.

\section{Angiotensin receptor neprilysin inhibitor}

The renin angiotensin-aldosterone system (RAAS) is a pivotal player in the pathophysiology of heart failure. ${ }^{10}$ Activation of this system is associated with activation of the sympathetic system and leads to vasoconstriction and sodium and water retention, ${ }^{11}$ with the degree of activation of this system correlating with the severity of heart failure. ${ }^{12}$ As a result, the focus of multiple heart failure trials has been to inhibit activation of the RAAS system and prevent its maladaptive consequences. The natriuretic peptide system, consisting of atrial natriuretic peptide and B-type natriuretic peptide (BNP), is commonly viewed as a counterweight to the RAAS system by promoting diuresis, natriuresis, and vasodilation. ${ }^{13}$ Neprilysin, an enzyme released by the kidney, is involved in the degradation of multiple vasoactive peptides, such as atrial natriuretic peptide, BNP, and bradykinin, which have all been shown to have protective effects in the heart failure syndrome. ${ }^{14}$ Given the harmful effects of neprilysin in heart failure, this endopeptidase has been the target of multiple landmark clinical trials (Figure 1).

The first trial examining the potential benefit of neprilysin inhibition was the Omapatrilat Versus Enalapril Randomized Trial of Utility in Reducing Events (OVERTURE trial ${ }^{15}$ ), which examined the combination of an ACE inhibitor and neprilysin inhibitor (omapatrilat) vs enalapril. In this study, there was no difference between the treatment groups relating to the primary outcome of a combined risk of death or heart failure hospitalization; however, secondary endpoint analysis showed a $9 \%$ decrease in cardiovascular death or hospitalization. This positive finding was offset by increased risk in the number and severity of episodes of angioedema, which was felt to be secondary to a marked increase in the circulating levels of bradykinin from dual neprilysin and ACE inhibition.

Given the safety concerns with omapatrilat, LCZ696, also known as sacubitril-valsartan, was developed. This agent combines an angiotensin receptor blocker-neprilysin inhibitor (ARNI) in hopes of reducing angioedema risk, as it inhibits only one of the enzymes responsible for bradykinin degradation. ${ }^{13}$ The earliest trial investigating this combination was an antihypertensive trial performed by Ruilope et al, ${ }^{16}$ where it was demonstrated that LCZ696 had larger antihypertensive effect compared to valsartan alone. Following this, the first heart failure trial using LCZ696, the Prospective comparison of ARNI with ARB on Management Of heart failure with preserved ejection fraction (PARAMOUNT trial ${ }^{17}$ ), examined the effect of this medication on the levels of N-terminal pro B-type natriuretic peptide (NT-ProBNP) in New York Heart Association (NYHA) class II-III patients with heart failure with preserved ejection fraction. This study demonstrated a greater reduction in NT-ProBNP at 12 weeks in the LCZ696 arm compared to the valsartan arm.

Most recently, results from the Prospective Comparison of ARNI with an Angiotensin-Converting Enzyme Inhibitor to Determine Impact on Global Mortality and Morbidity in Heart Failure (PARADIGM-HF) trial ${ }^{18}$ have been released. This trial is the largest randomized controlled trial in heart failure. PARADIGM-HF examined the effects of LCZ696 compared to those of enalapril on 8442 patients with heart failure and reduced ejection fraction. The primary outcome measure was cardiovascular death or hospitalization for heart failure. Patients enrolled were NYHA class II-IV (predominantly classes II and III) with an ejection fraction of $\leq 40 \%$. The unique design of this trial included three phases: a single-blind run in a phase where all patients received enalapril, followed by a single-blind run in a phase where all patients received LCZ696, followed by the double-blind treatment phase. This three-phase approach was performed 


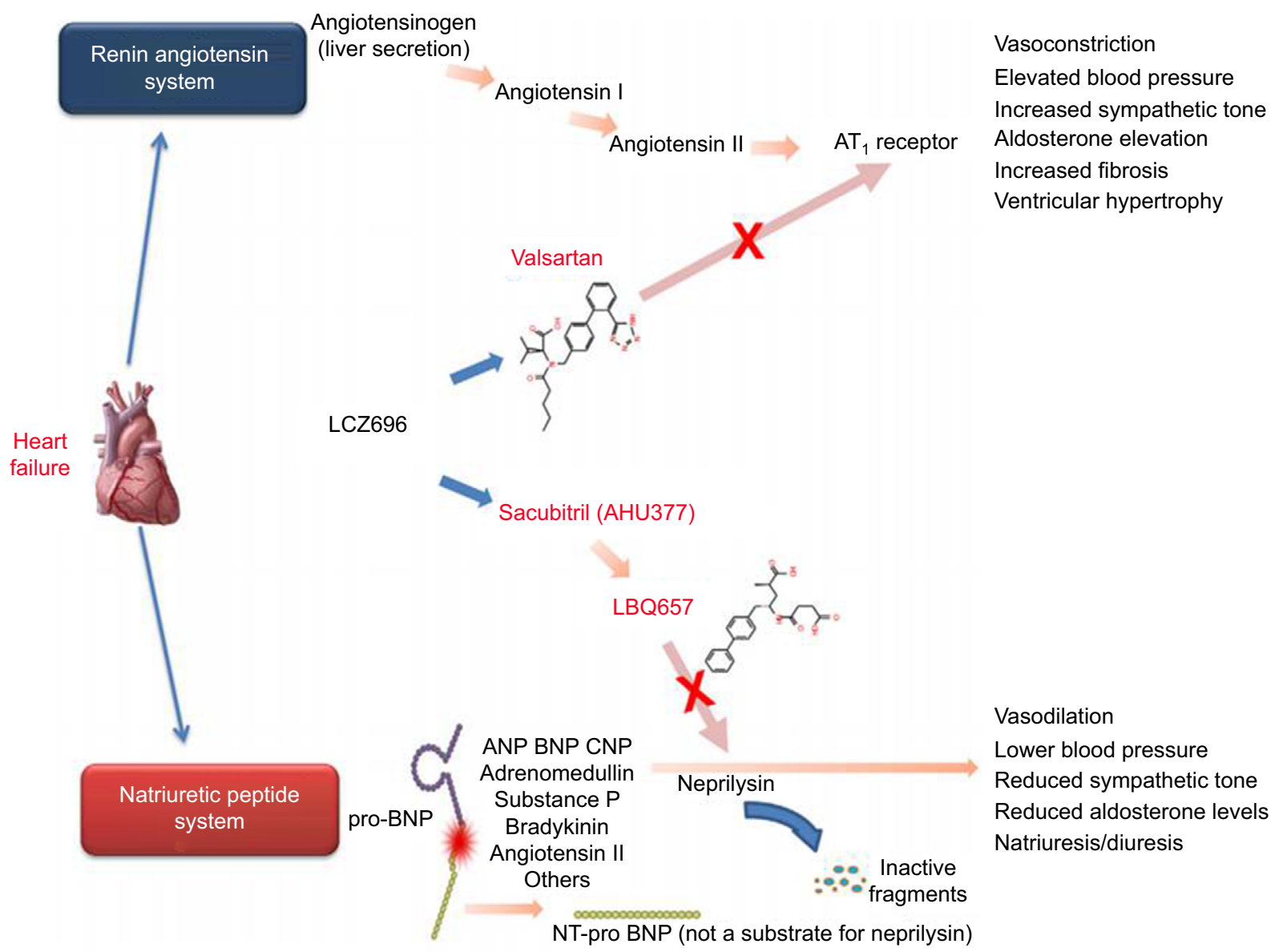

Figure I Mechanism of action of LCZ696.

Notes: LCZ696 is composed of valsartan (an angiotensin receptor blocker) and sacubitril (a neprilysin inhibitor). Valsartan prevents the binding of angiotensin II to the angiotensin receptor and results in vasodilation, decreased sympathetic tone, and decreased levels of aldosterone. Inhibition of neprilysin by sacubitril prevents the breakdown of both ANP and BNP, resulting in vasodilation, diuresis, natriuresis, and reduced aldosterone levels. Reprinted from JACC: Heart failure; 2(6). Vardeny O, Miller R, Solomon SD. Combined neprilysin and renin-angiotensin system inhibition for the treatment of heart failure. 663-670. Copyright () 2014 American College of Cardiology Foundation. Published by Elsevier Inc. All rights reserved. With permission from Elsevier. ${ }^{13}$

Abbreviations: ANP, atrial natriuretic peptide; $\mathrm{AT}_{1}$, angiotensin I; BNP, B-type natriuretic peptide; CNP, c-type natriuretic peptide; NT, N-terminal.

to ensure that patients randomized would be able to tolerate the drugs as well as their side effects at target doses during the trial (LCZ696 target $400 \mathrm{mg}$ /day, enalapril $20 \mathrm{mg}$ /day).

In PARADIGM-HF, recipients of LCZ696 had a $20 \%$ relative reduction in the composite outcome of cardiovascular death or heart failure hospitalization, compared to enalapril recipients (Figure 2). When cardiovascular death and heart failure hospitalizations were viewed as separate outcomes, LCZ696 also conferred a 20\% relative reduction in both. From a safety standpoint, patients on LCZ696 were more likely to experience hypotension and nonserious angioedema, while those on enalapril were more likely to have renal impairment, hyperkalemia, and cough. Furthermore, a subanalysis of the PARADIGM-HF trial ${ }^{19}$ showed a $38 \%$ reduction in 30-day readmission for patients on LCZ696. In addition, an analysis of the mode of death in PARADIGM-HF revealed a significant reduction with LCZ696 compared to enalapril in the risk of both sudden cardiac death as well as death from worsening congestive heart failure. ${ }^{20}$
Based on the findings from PARADIGM, the American College of Cardiology, the American Heart Association, and the Heart Failure Society of America released a consensus statement ${ }^{21}$ regarding the use of a dual angiotensin-neprilysin inhibitor (sacubitril-valsartan) for patients with heart failure and reduced systolic function. In this statement, they recommend the initiation of sacubitrilvalsartan in patients with mild to moderate heart failure (defined as a BNP $\geq 150 \mathrm{pg} / \mathrm{mL}$ or NT-proBNP $\geq 600 \mathrm{pg}$ / $\mathrm{mL}$ or a $\mathrm{BNP} \geq 100 \mathrm{pg} / \mathrm{mL}$ or NT-proBNP $\geq 400 \mathrm{pg} / \mathrm{mL}$ in the setting of a heart failure admission within the last 12 months) who are able to tolerate an equivalent of enalapril $10 \mathrm{mg}$ twice a day (class I, level of evidence B) to further reduce cardiovascular mortality or heart failure admissions. Additionally, they emphasized that sacubitril-valsartan should not be given concomitantly with an ACE inhibitor or within 36 hours of an ACE inhibitor to minimize the risk of angioedema and that it is contraindicated in patients with a history of angioedema. 
A

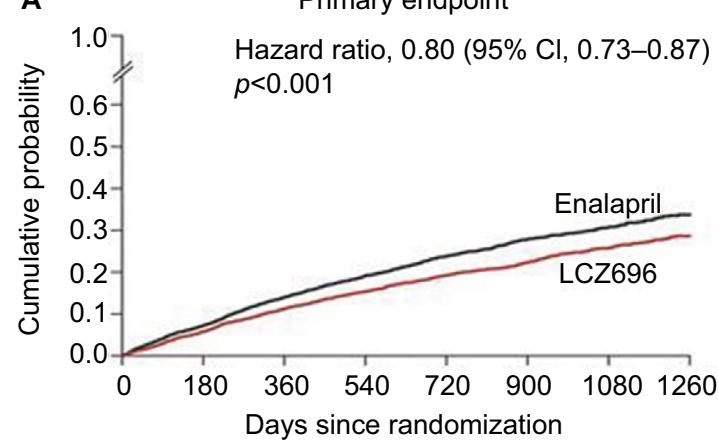

No. at risk

$\begin{array}{lllllllll}\text { LCZ696 } & 4187 & 3922 & 3663 & 3018 & 2257 & 1544 & 896 & 249\end{array}$

Enalapril $\begin{array}{lllllllll}4212 & 3883 & 3579 & 2922 & 2123 & 1488 & 853 & 236\end{array}$

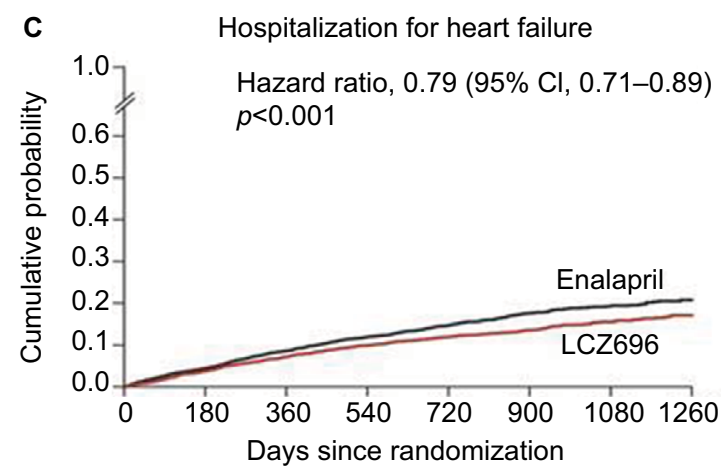

No. at risk

$\begin{array}{lllllllll}\text { LCZ696 } & 4187 & 3922 & 3663 & 3018 & 2257 & 1544 & 896 & 249\end{array}$

$\begin{array}{lllllllll}\text { Enalapril } & 4212 & 3883 & 3579 & 2922 & 2123 & 1488 & 853 & 236\end{array}$

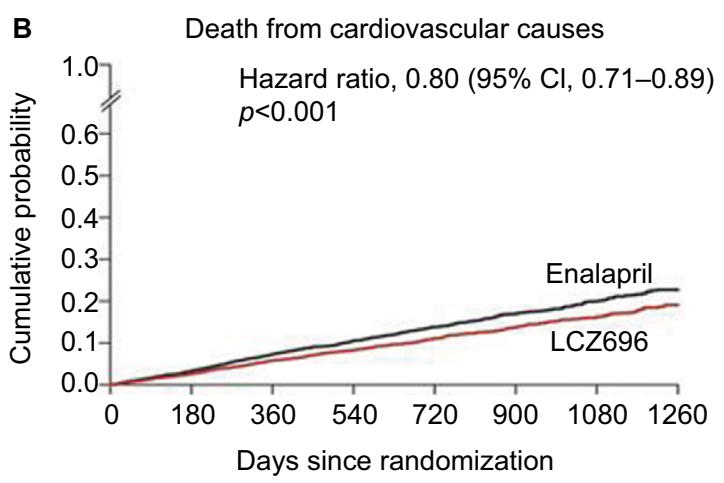

No. at risk

$\begin{array}{lllllllll}\text { LCZ696 } & 4187 & 4056 & 3891 & 3282 & 2478 & 1716 & 1005 & 280\end{array}$

$\begin{array}{lllllllll}\text { Enalapril } & 4212 & 4051 & 3860 & 3231 & 2410 & 1726 & 994 & 279\end{array}$

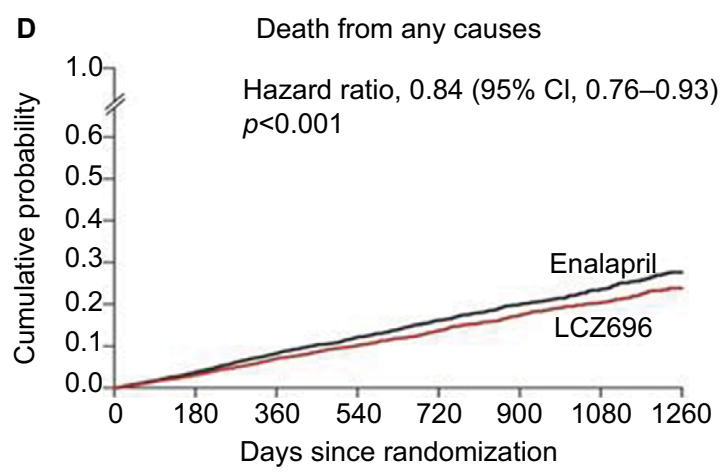

No. at risk

LCZ696 $44187 \quad 4056 \quad 389132822478 \quad 1716 \quad 1005 \quad 280$

$\begin{array}{lllllllll}\text { Enalapril } & 4212 & 4051 & 3860 & 3231 & 2410 & 1726 & 994 & 279\end{array}$

Figure 2 Kaplan-Meier curves for key study outcomes in the PARADIGM-HF trial.

Notes: (A) Primary end point. (B) Death from cardiovascular causes. (C) Hospitalization for heart failure. (D) Death from any causes. From New England Journal of Medicine. McMurray JJ, Packer M, Desai AS, et al. Angiotensin-Neprilysin Inhibition versus Enalapril in Heart Failure. 37I(II):993-I004. Copyright (C 20I4 Massachusetts Medical Society. Reprinted with permission from Massachusetts Medical Society. ${ }^{18}$

Abbreviations: $\mathrm{Cl}$, confidence interval; PARADIGM-HF, Prospective Comparison of ARNI with an Angiotensin-Converting Enzyme Inhibitor to Determine Impact on Global Mortality and Morbidity in Heart Failure.

\section{Ivabradine}

Elevated heart rate has been shown to be a risk factor for adverse outcomes in patients with cardiovascular diseases such as hypertension, ${ }^{22}$ coronary artery disease, ${ }^{23}$ and congestive heart failure. ${ }^{24}$ The cause is thought to be multifactorial, including increased oxygen demand and consumption, decreased ventricular efficiency, development of tachycardiainduced cardiomyopathies, decreased coronary perfusion, and increased cardiac hypertrophy ${ }^{25}$ (Figure 3). Classes of medications such as beta blockers and calcium channel blockers can decrease the heart rate; however, their use is limited by their negative ionotropic effects, intolerances, as well as tachycardia, despite maximal doses.

Located in the pacemaker cells of the heart is the funny current $\left(\mathrm{I}_{\mathrm{f}}\right)$, a mixed sodium-potassium current whose activation and binding to the funny channel is responsible for the initiation of diastolic depolarization. Inhibition of this current increases diastole and results in decreased heart rate.
Ivabradine is a specific inhibitor of $\left(\mathrm{I}_{\mathrm{f}}\right)$ within the sinoatrial node, and, given its specificity, is thought to be able to decrease the heart rate without altering myocardial contractility or blood pressure. ${ }^{26,27}$ This hypothesis was evaluated in the Ivabradine and Outcomes in Chronic Heart Failure (SHIFT trial $^{28}$ ), which compared the effect of ivabradine vs placebo in patients with symptomatic heart failure with an ejection fraction of $\leq 35 \%$ and a heart rate of 70 beats per minute or higher, who had been admitted to the hospital for heart failure within the previous year and were on stable background therapy including a beta blocker if tolerated. The primary outcome of this study was a composite of cardiovascular death or hospital admission for heart failure. After a median follow-up of nearly 23 months, there was an $18 \%$ decrease in the primary outcome, which was driven predominantly by a decrease in heart failure readmission. The findings in this study are limited by the fact that only $25 \%$ of patients within this trial were on optimal doses of beta blockade. 


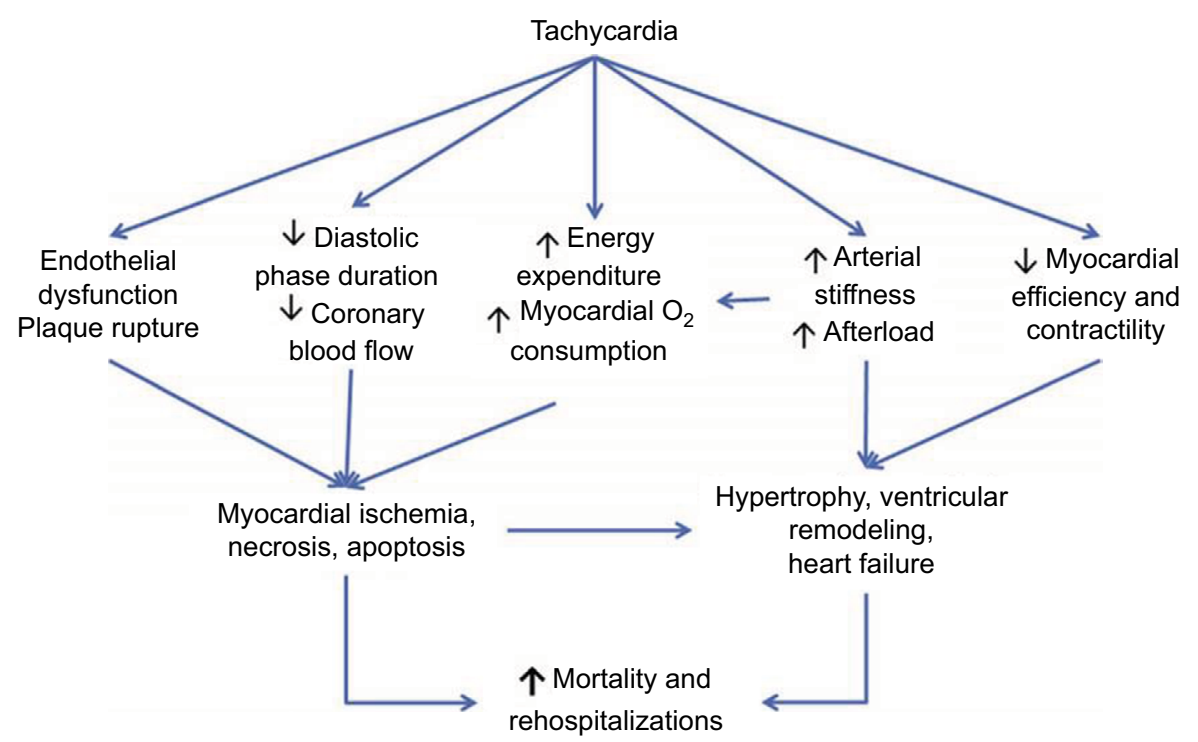

Figure 3 Mechanism of tachycardia and increased cardiovascular morbidity and mortality.

Notes: The cause of increased morbidity and mortality with tachycardia in cardiovascular disease is multifactorial. Reprinted from JACC: Heart Failure. I(6). Metra M. Tachycardia after a heart failure hospitalization: another piece of the puzzle? 497-499. () 2013 by the American College of Cardiology Foundation. Published by Elsevier Inc. With permission from American College of Cardiology Foundation. ${ }^{56}$

Based on this study, the American College of Cardiology/American Heart Association/Heart Failure Society of America recommends the use of ivabradine to reduce heart failure hospitalizations in patients with symptomatic, NYHA class II-III heart failure with an ejection fraction of $\leq 35 \%$, who are in sinus rhythm, with a heart rate of $\geq 70$ beats per minute at rest despite a maximally tolerated dose of beta blocker (class IIa, level of evidence B).

\section{Hemodynamic monitoring}

By the year 2030, the predicted cost of heart failure will be $>70$ billion US dollars, with $80 \%$ of the cost attributed to costs associated with hospitalizations. ${ }^{7}$ Moreover, $50 \%$ of patients admitted to the hospital for heart failure management do not survive beyond 3 years. ${ }^{29}$ Given these findings, decreasing readmission rates is considered a major opportunity to improve both the costs and outcomes associated with the care of patients with heart failure. In 2010, the Patient Protection and Affordable Care Act directed the Center for Medicare and Medicaid Services to penalize hospitals with worse than expected 30-day readmission rates, with penalties of up to $1 \%$ in $2013,2 \%$ in 2014 , and $3 \%$ in $2016 .{ }^{30}$

Strategies to reduce recurrent heart failure hospitalizations have focused on three distinct time frames: before discharge, early after discharge, and late after discharge.$^{19}$ Prior to discharge from the hospital, focus is placed on ensuring that patients are adequately decongested as well as identifying and preemptively controlling triggers that may lead to potential readmission, such as medical noncompliance, dietary indiscretion, or lack of social support. Decongestion remains the primary area of focus, given that patients with high level of congestion at discharge are 15 times more likely to be readmitted or die compared to those who have achieved complete decongestion..$^{31}$ Despite the fact that $60 \%$ of patients leave the hospital with no or a minimal level of congestion, by 60 days, $40 \%$ of these patients are recongested. ${ }^{32}$ In the early discharge phase, focus is on patient education, medication reconciliation, and early contact/follow-up. Unfortunately, interventions in this phase have shown inconsistent results in their ability to decrease readmissions or improve mortality. ${ }^{33,34}$ Telemonitoring has been a strategy that has been extensively studied in the late after discharge phase. The potential benefit of telemonitoring relates to the fact that the majority of days that patients with heart failure receive care are usually with direct contact with the health care system, and that information transmitted, such as daily weights, symptoms, and vital signs, may allow physicians to intervene and prevent patients from being readmitted to the hospital. Unfortunately, three separate trials $\mathrm{s}^{35-37}$ failed to demonstrate any improvement in the reduction of cardiovascular death or readmission. The lack of benefit seen in these trials may be because measured variables such as weight gain might be good surrogates for congestion in the short term, but may not be good surrogates in the long term. Also, management responses to a change in the variables measured were not standardized and were left up to individual provider's preference.

Studies examining physiologic changes that precede admissions for acute decompensated heart failure have shown 
that the first change noted is an increase in filling pressure which can occur up to 20 days prior to admission, followed by changes in autonomic adaptation and intrathoracic impedance, and finally weight gain and the development of symptoms occurring only a few days prior to admission. ${ }^{38}$ Given the potential importance of antecedent detection of elevated intracardiac filling pressures, there has been great interest in developing high-fidelity implantable pulmonary artery (PA) pressure measurement systems. The most well-studied PA measurement system is the CardioMems heart failure sensor, a wireless, implantable hemodynamic monitoring system consisting of a coil and a pressure-sensitive capacitor encased in a hermetically sealed silica capsule covered by silicone which anchors into the PA using nitinol loops ${ }^{39}$ (Figure 4). Patients can transmit hemodynamic data remotely using this wireless device, with PA pressure tracing data then reviewed by their heart failure clinicians, who then interpret the information and prompt changes in medical therapy.

The CardioMems device was originally examined in the CHAMPION trial, ${ }^{40}$ which examined the 6-month rate of heart failure-related admissions in patients with NYHA functional class III symptoms and a heart failure hospitalization in the past 12 months. All patients in this study received the CardioMems device; however, half of the patients were put into the treatment arm, in which a clinician was given access to their PA pressure readings obtained by the device, while the remaining patients were in the control group and a clinician did not have access to their device readings. Patients in the treatment arm of this trial demonstrated a $28 \%$ decrease in heart failure-related hospitalizations over the 6-month time periods and a $37 \%$ decrease during the entire follow-up period (Figure 5). Additional findings from this study demonstrated that patients in the treatment arm had a lower risk of death or first heart failure-related hospitalization, a greater reduction in PA mean pressure, more days spent outside the hospital, and a better quality of life. Interestingly, when readmitted for heart failure, patients in the treatment arm had a 2-day shorter length of stay than the control group. A unique finding in this trial was that the benefits displayed occurred in both patients with preserved and reduced systolic function. A follow-up study of the CHAMPION trial ${ }^{41}$ examined findings of the open access period during which time data from all patients remaining in the trial were accessible to clinicians. Patients originally in the treatment arm had a sustained decrease in their rate of readmission, while those who were originally in the control arm and now had their data given to clinicians showed a $48 \%$ decrease in their rates of readmission to the hospital for heart failure.

Based on the results of the original CHAMPION study, in 2014, the CardioMems hemodynamic monitoring device was approved by the US Food and Drug Administration for use in NYHA class III heart failure patients who have been hospitalized for heart failure in the past 12 months, with the goal of decreasing readmissions. Given the requirement of review of the transmitted data by a health care provider, this device must be embedded within a robust heart failure disease management program to have a real impact.

\section{Heart-assist devices}

Ever since the development of the artificial heart program in 1964, multiple different mechanical circulatory support devices have been developed and tested in advanced stage heart failure patients for both short-term and long-term use. Early-generation LVADs were pulsatile in flow, and despite their US Food and Drug Administration approval in 2002, were rarely used secondary to their large size, limited durability, high rate of device failure, and serious complications such as stroke and infection. ${ }^{42}$ Since that time, there have been rapid technological improvements resulting in the development of continuous flow LVADs, which are smaller, valveless, and consist of only a single moving internal rotor. Studies have shown that continuous flow LVADs have improved durability and survival, along with less morbidity. ${ }^{43}$ At the current time, there are two different types of continuous flow pumps: axial ${ }^{44}$ and centrifugal ${ }^{45}$ (Box 1). ${ }^{46}$

The HeartMate II (HMII) LVAD, the most commonly implanted axial flow device, consists of an inflow cannula arising from the left ventricle, an inlet stator and blood flow straightener, a rotor, an outlet stator and diffuser, and an outflow cannula that connects to the aorta. ${ }^{43}$ Within this device, the rotor is suspended with mechanical bearings on spherical surfaces that rotate in sockets. Given this, the bearing contact points are predisposed to mechanical wear and heat generation, which may theoretically limit the operating life span of the pump. Also, the increase in wear and heat generation at the bearing contact points increases the chances of fibrin deposition and thrombus formation in the blood flow path. ${ }^{47}$ In contrast, the HeartWare LVAD, the most commonly implanted centrifugal flow device, has an impeller that is suspended by passive magnetic and hydrodynamic thrust bearings to allow for a contact-free rotation, with the hope of decreasing device thrombosis and resultant pump exchange. ${ }^{48}$ However, despite these technological advances, $20 \%$ of patients will experience major bleeding at 1 year, $10 \%$ of patients will suffer a disabling stroke, $6 \%-8 \%$ will experience pump thrombosis, and $20 \%$ of patients will experience a serious device-related infection. ${ }^{49}$ Given the high rate of morbidity, the focus on recent LVAD research has been to improve outcomes in these three specific areas. 
A

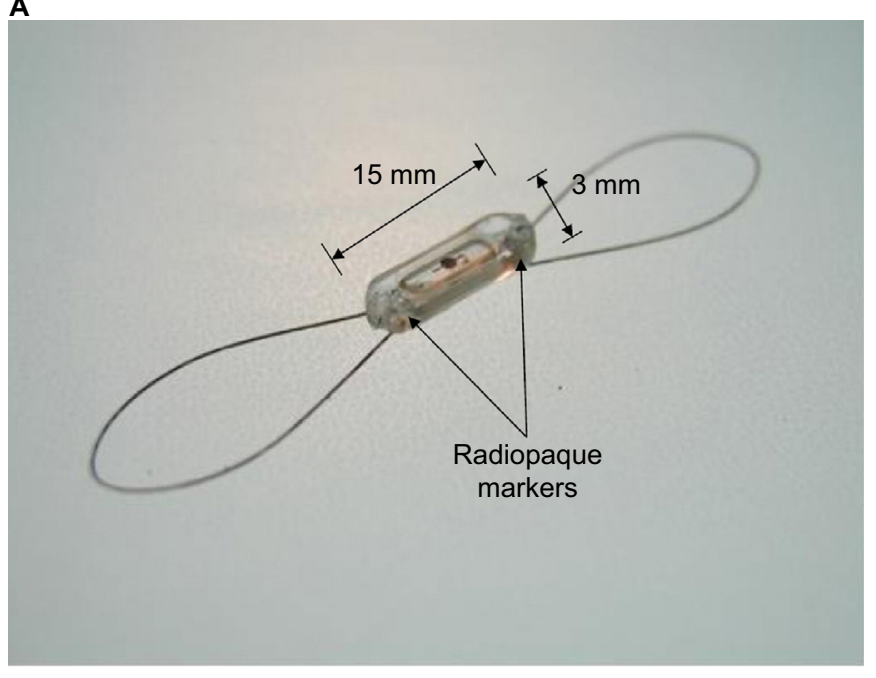

B

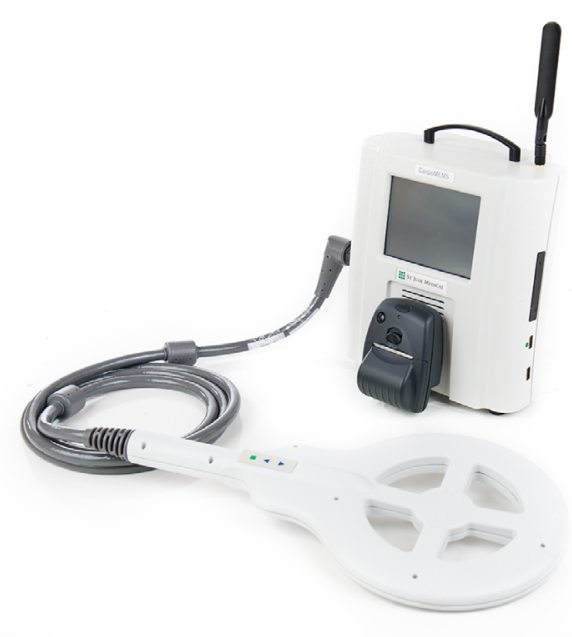

C

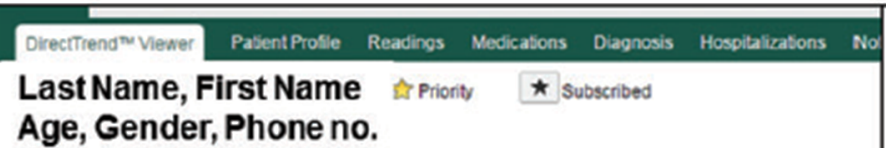

\section{Fixed Auto}

From: $10-12 \cdot 2014$ To: $01-30-2016$

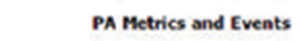

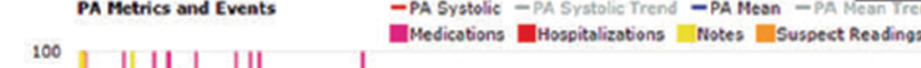

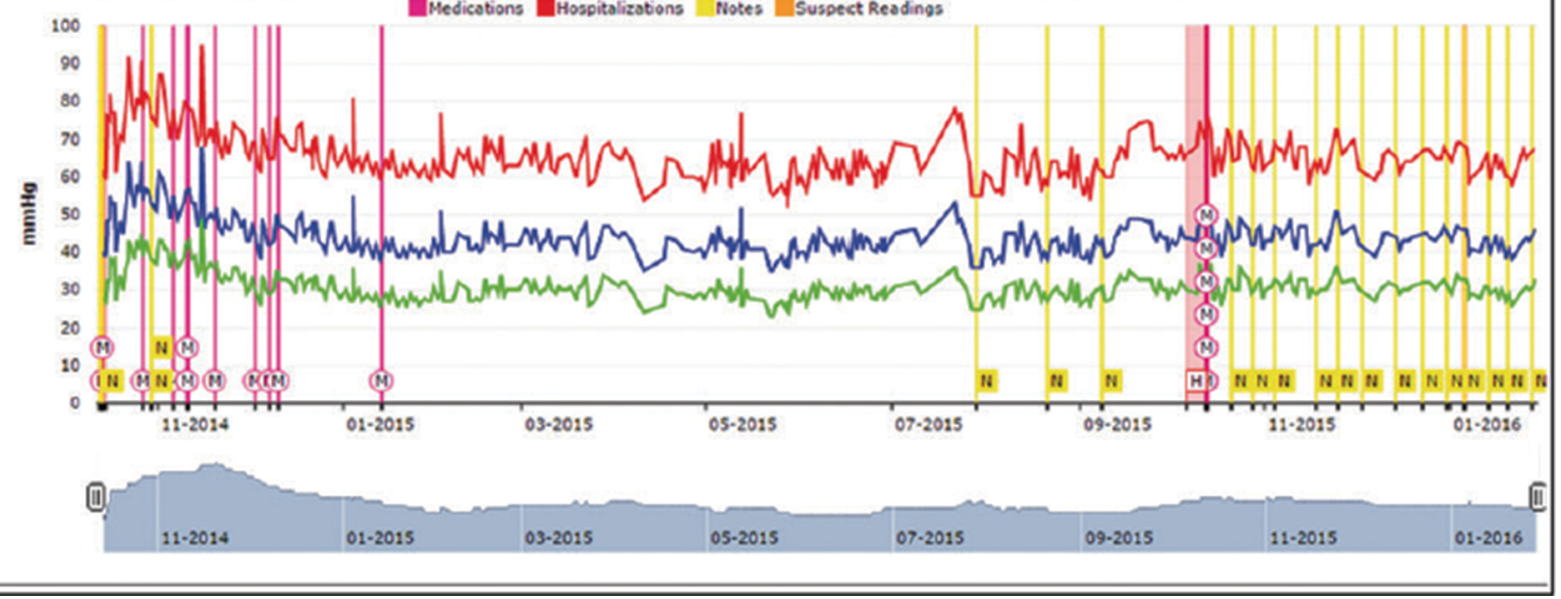

Figure 4 CardioMems device.

Notes: The CardioMems system consists of three entities: (A) a PA sensor used to measure the PA diastolic pressure, (B) a home electronics system which both powers the PA sensor and transmits information to the third part, and (C) a centralized database accessible to a health care provider. Figure $\mathbf{A}$ reprinted from Heart Lung Circulation; 2 I (6-7). Crozier I, Smith W. Modern device technologies. 320-327. Copyright (C) 20II Australian and New Zealand Society of Cardiac and Thoracic Surgeons (ANZSCTS) and the Cardiac Society of Australia and New Zealand (CSANZ), with permission from ANZSCTS and CSANZ. ${ }^{57}$ Figure B - CardioMems ${ }^{\mathrm{TM}}$ hospital electronics system: CardioMems and St. Jude Medical are trademarks of St. Jude Medical, LLC or its related companies. Reproduced with permission of St. Jude Medical, @20I7. All rights reserved. Figure $\mathbf{C}$ reprinted from Shavelle D, Jermyn R. The CardioMEMS heart failure sensor: a procedural guide for implanting physicians. The Journal of Invasive Cardiology. 2016;28(7):273-279.58

Abbreviation: PA, pulmonary artery.

A next-generation heart-assist device undergoing active investigation is the HeartMate 3 (HM3), which was developed to address the common adverse outcomes in the current generation of devices, including bleeding, infection, stroke, and pump thrombosis, by improving hemocompatibility. This device has several novel design elements. A fully magnetically levitated rotor eliminates the need for mechanical or hydrodynamic bearings producing blood gaps, which are 10-20 times wider than the current devices, and the device can be programmed to facilitate rapid changes in the rotor speed to create an intrinsic artificial pulse, washing the pump circuit (Figure 6). In combination, these features are hypothesized to decrease damage 


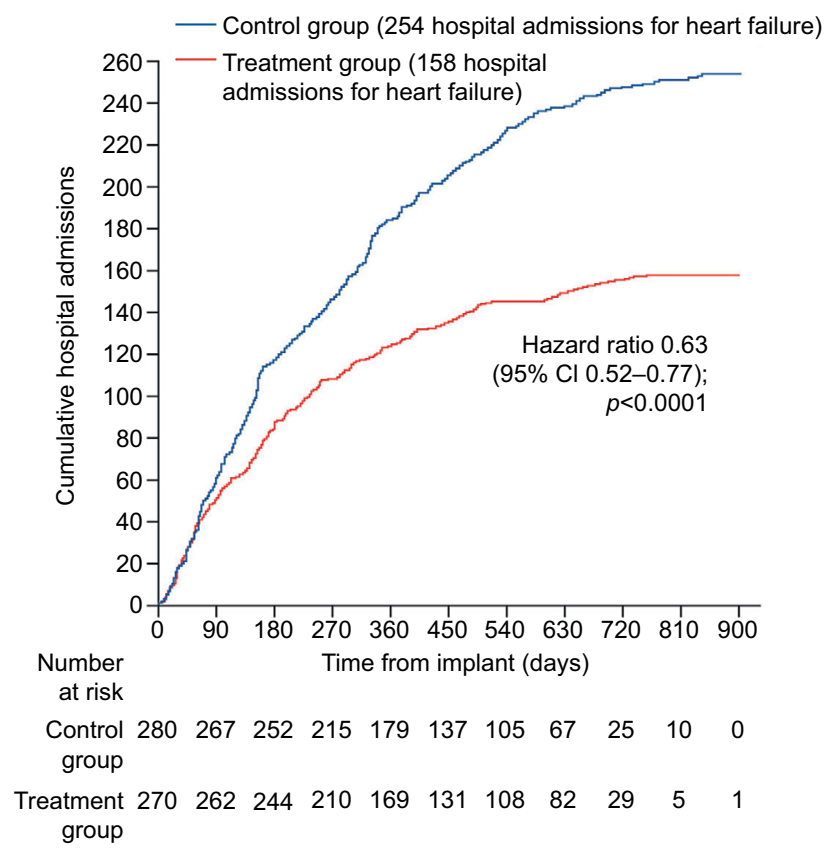

Figure 5 Impact of CardioMems on heart failure readmissions.

Notes: Kaplan-Meier curve for heart failure-related hospitalization. Reprinted from The Lancet; 377(9766). Abraham WT, Adamson PB, Bourge RC, et al. Wireless pulmonary artery haemodynamic monitoring in chronic heart failure: a randomised controlled trial. 658-666. Copyright 20II. With permission from Elsevier. ${ }^{40}$ Abbreviation: $\mathrm{Cl}$, confidence interval.

\section{Box I Triggers for referral for VAD evaluation}

Inability to wean inotropes or frequent inotrope use
Peak VO2 $<14-16 \mathrm{~mL} \cdot \mathrm{kg}^{-1} \cdot \mathrm{min}^{-1}$ or $<50 \%$ predicted
Two or more HF admissions in 12 mo
Worsening right heart failure and secondary pulmonary hypertension
Diuretic refractoriness associated with worsening renal function
Circulatory-renal limitation to ACE inhibition
Hypotension limiting $\beta$-blocker therapy
NYHA class IV symptoms at rest on most days
Seattle HF model score with anticipated mortality $>I 5 \%$ at I y
Six-minute walk distance $<300$ m
Persistent hyponatremia (serum sodium $<134$ mEq/L)
Recurrent, refractory ventricular tachyarrhythmias
Cardiac cachexia

Note: Reproduced from Stewart and Givertz. Stewart GC, Givertz MM. Mechanical circulatory support for advanced heart failure: patients and technology in evolution. Circulation. 2012;125(10):1304-1315. Copyright (c) 2012 American Heart Association, Inc. http://circ.ahajournals.org/. Promotional and commercial use of the material in print, digital or mobile device format is prohibited without the permission from the publisher Wolters Kluwer. Please contact healthpermissions@wolterskluwer.com for further information. ${ }^{46}$

Abbreviations: ACE, angiotensin-converting enzyme; HF, heart failure; mo, months; NYHA, New York Heart Association; VAD, ventricular assist device; $\mathrm{VO}_{2}$, oxygen consumption; $y$, year.

to red blood cells and von Willebrand factor, decrease platelet activation, and reduce stasis in the pump with the end goals of decreasing strokes, bleeding, and pump thrombosis. ${ }^{50-52}$ Additional differences between the HM3 and HMII devices include a largely intrapericardial placement rather than subdiaphragmatic position, as well as a longer battery life.
The first clinical study of the HM3 was performed by Netuka et al ${ }^{52}$ in its Conformité Européenne CE Mark clinical trial and examined the 6-month outcomes in patients implanted with this device in ten centers in six countries. Patients included in this study were either NYHA class IIIb (52\%) or NYHA class IV ( $48 \%$ ), with $54 \%$ being a bridge to therapy candidate and $46 \%$ being a destination therapy candidate. By 6 months, $88 \%$ of patients were alive on the device, $4 \%$ had been transplanted, and $8 \%$ died. Remarkably, the 30-day postimplant survival rate was $98 \% .{ }^{53}$ Key adverse events at 6 months included bleeding (14\%), driveline infection (10\%), and debilitating stroke (8\%). Most impressively, there were no reported cases of hemolysis or pump thrombosis within the study period. The preliminary outcomes and adverse event profiles from the HM3 experience were as good or better than those of previous trials evaluating the HMII and HeartWare devices. ${ }^{44,45,54}$

After much anticipation, the results of the short-term support analysis of the MOMENTUM 3 trial were released in November 2016. This trial examined 294 patients who were randomized in a 1:1 fashion to either the HMII or HM3 device with a primary composite endpoint of survival free of disabling stroke or survival free of reoperation for device replacement or removal at 6 months ${ }^{55}$ Patients in this trial were included irrespective of their candidacy for heart transplantation. Although powered for noninferiority, analysis of the primary endpoint showed superiority of the HM3 $(86.2 \%$ vs $76.8 \%, p=0.04)$. This endpoint was largely driven by the absence of confirmed or suspected pump thrombosis in the HM3, which was present in $10.1 \%$ of patients with the axial flow device. Despite the improvement in pump thrombosis, there was no difference between the devices in relation to other device complications such as right heart failure, stroke (any or disabling), infections, or bleeding. The MOMENTUM 3 trial will next report the randomized long-term support cohort $(\mathrm{N}=366)$ for the composite endpoint of survival free of disabling stroke or survival free of reoperation for device replacement or removal at 24 months. Thanks to its adaptive design, the MOMENTUM 3 trial is now fully enrolled with 1028 subjects and powered to evaluate a secondary endpoint of pump replacement at 2 years. ${ }^{50}$

\section{Conclusion}

The last 10 years have been a renaissance for the treatment of the heart failure syndrome with the development of new pharmacologic therapies, hemodynamic monitoring devices, and left ventricular assist systems that have been shown to improve morbidity and mortality as well as decrease heart failure readmissions. Studies examining these newly 


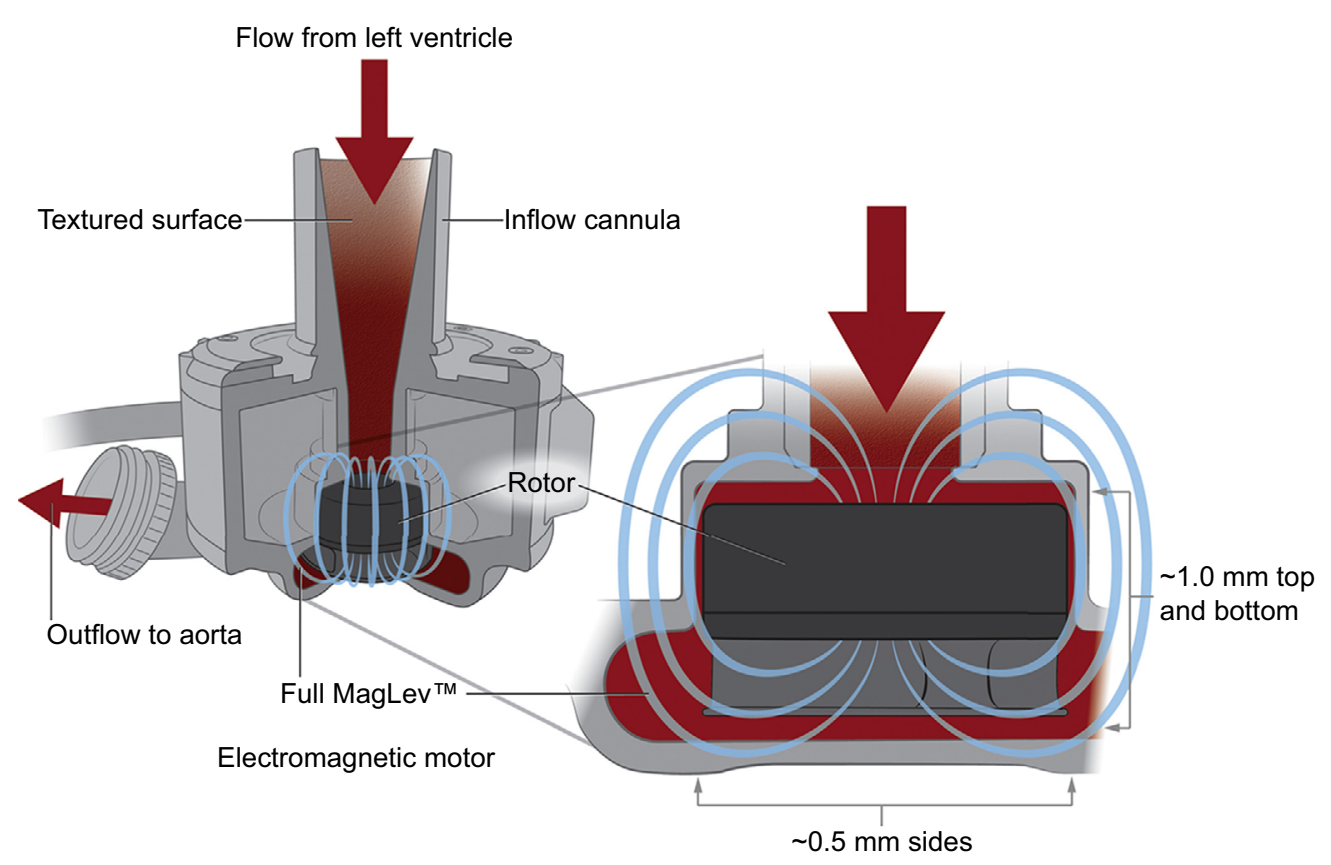

Figure 6 HeartMate 3 Full MagLev ${ }^{\top M}$ internal pump design.

Notes: Reprinted from Journal of the American College of Cardiology; 66(23). Netuka I, Sood P, Pya Y, et al. Fully Magnetically Levitated Left Ventricular Assist System for Treating Advanced HF: A Multicenter Study. 2579-2589. Copyright @ 2015 American College of Cardiology Foundation. Published by Elsevier Inc. All rights reserved. With permission of Elsevier. ${ }^{52}$

discussed therapies as well as trials examining novel therapeutic agents are ongoing, with hopes of future discoveries to help treat this disease.

\section{Disclosure}

The authors report no conflicts of interest in this work.

\section{References}

1. Yancy CW, Jessup M, Bozkurt B, et al. 2013 ACCF/AHA guideline for the management of heart failure: a report of the American College of Cardiology Foundation/American Heart Association Task Force on Practice Guidelines. J Am Coll Cardiol. 2013;62(16):e147-e239.

2. Writing Group M, Mozaffarian D, Benjamin EJ, et al; American Heart Association Statistics Committee; Stroke Statistics Subcommittee. Heart disease and stroke statistics-2016 update: a report from the American Heart Association. Circulation. 2016;133(4):e38-e360.

3. Bui AL, Horwich TB, Fonarow GC. Epidemiology and risk profile of heart failure. Nat Rev Cardiol. 2011;8(1):30-41

4. Hall MJ, DeFrances CJ, Williams SN, Golosinskiy A, Schwartzman A. National hospital discharge survey: 2007 summary. Nat Health Stat Report. 2010;(29):1-20, 24.

5. Anne Staylor RN, MS. Heart failure therapy: past, present, and future. 2002. Available from: http://www.medscape.org/viewarticle/433746. Accessed November 1, 2016.

6. Roger VL, Weston SA, Redfield MM, et al. Trends in heart failure incidence and survival in a community-based population. JAMA. 2004;292(3): 344-350.

7. Heidenreich PA, Albert NM, Allen LA, et al. Forecasting the impact of heart failure in the United States: a policy statement from the American Heart Association. Circ Heart Fail. 2013;6(3):606-619.

8. Cohn JN, Archibald DG, Ziesche S, et al. Effect of vasodilator therapy on mortality in chronic congestive heart failure. Results of a Veterans Administration Cooperative Study. N Engl J Med. 1986;314(24):1547-1552.
9. Fonarow GC, Yancy CW, Hernandez AF, Peterson ED, Spertus JA, Heidenreich PA. Potential impact of optimal implementation of evidencebased heart failure therapies on mortality. Am Heart J. 2011;161(6): 1024-1030. e1023.

10. Verbrugge FH, Tang WH, Mullens W. Renin-angiotensin-aldosterone system activation during decongestion in acute heart failure: friend or foe? JACC Heart Fail. 2015;3(2):108-111.

11. Jhund PS, McMurray JJ. The neprilysin pathway in heart failure: a review and guide on the use of sacubitril/valsartan. Heart. 2016;102(17): 1342-1347.

12. Mentz RJ, Stevens SR, DeVore AD, et al. Decongestion strategies and renin-angiotensin-aldosterone system activation in acute heart failure. JACC Heart Fail. 2015;3(2):97-107.

13. Vardeny O, Miller R, Solomon SD. Combined neprilysin and reninangiotensin system inhibition for the treatment of heart failure. JACC Heart Fail. 2014;2(6):663-670.

14. Bayes-Genis A. Neprilysin in heart failure: from oblivion to center stage. JACC Heart Fail. 2015;3(8):637-640.

15. Packer M, Califf RM, Konstam MA, et al. Comparison of omapatrilat and enalapril in patients with chronic heart failure: the Omapatrilat Versus Enalapril Randomized Trial of Utility in Reducing Events (OVERTURE). Circulation. 2002;106(8):920-926.

16. Ruilope LM, Dukat A, Bohm M, Lacourciere Y, Gong J, Lefkowitz MP. Blood-pressure reduction with LCZ696, a novel dual-acting inhibitor of the angiotensin II receptor and neprilysin: a randomised, doubleblind, placebo-controlled, active comparator study. Lancet. 2010; 375(9722):1255-1266.

17. Solomon SD, Zile M, Pieske B, et al. The angiotensin receptor neprilysin inhibitor LCZ696 in heart failure with preserved ejection fraction: a phase 2 double-blind randomised controlled trial. Lancet. 2012; 380(9851):1387-1395.

18. McMurray JJ, Packer M, Desai AS, et al; PARADIGM-HF Investigators and Committees. Angiotensin-neprilysin inhibition versus enalapril in heart failure. N Engl J Med. 2014;371(11):993-1004.

19. Desai AS. Intensive management to reduce hospitalizations in patients with heart failure. Circulation. 2016;133(17):1704-1707. 
20. Desai AS, McMurray JJ, Packer M, et al. Effect of the angiotensinreceptor-neprilysin inhibitor LCZ696 compared with enalapril on mode of death in heart failure patients. Eur Heart J. 2015;36(30):1990-1997.

21. Yancy CW, Jessup M, Bozkurt B, et al. 2016 ACC/AHA/HFSA focused update on new pharmacological therapy for heart failure: an update of the 2013 ACCF/AHA guideline for the management of heart failure: a report of the American College of Cardiology/American Heart Association Task Force on Clinical Practice Guidelines and the Heart Failure Society of America. Circulation. 2016;134(13):e282-e293.

22. Gillman MW, Kannel WB, Belanger A, D'Agostino RB. Influence of heart rate on mortality among persons with hypertension: the Framingham Study. Am Heart J. 1993;125(4):1148-1154.

23. Fox K, Ford I, Steg PG, Tendera M, Robertson M, Ferrari R; Beautiful investigators. Heart rate as a prognostic risk factor in patients with coronary artery disease and left-ventricular systolic dysfunction (BEAUTIFUL): a subgroup analysis of a randomised controlled trial. Lancet. 2008;372(9641):817-821.

24. Pocock SJ, Wang D, Pfeffer MA, et al. Predictors of mortality and morbidity in patients with chronic heart failure. Eur Heart $J$. 2006;27(1):65-75.

25. Perret-Guillaume C, Joly L, Benetos A. Heart rate as a risk factor for cardiovascular disease. Prog Cardiovasc Dis. 2009;52(1):6-10.

26. DiFrancesco D. Funny channels in the control of cardiac rhythm and mode of action of selective blockers. Pharmacol Res. 2006;53(5): 399-406.

27. DiFrancesco D, Borer JS. The funny current: cellular basis for the control of heart rate. Drugs. 2007;67(Suppl 2):15-24.

28. Swedberg K, Komajda M, Bohm M, et al. Ivabradine and outcomes in chronic heart failure (SHIFT): a randomised placebo-controlled study. Lancet. 2010;376(9744):875-885.

29. Barker WH, Mullooly JP, Getchell W. Changing incidence and survival for heart failure in a well-defined older population, 1970-1974 and 1990-1994. Circulation. 2006;113(6):799-805.

30. Bradley EH, Curry L, Horwitz LI, et al. Hospital strategies associated with 30-day readmission rates for patients with heart failure. Circ Cardiovasc Qual Outcomes. 2013;6(4):444-450.

31. Logeart D, Thabut G, Jourdain P, et al. Predischarge B-type natriuretic peptide assay for identifying patients at high risk of re-admission after decompensated heart failure. $J$ Am Coll Cardiol. 2004;43(4): 635-641.

32. Lala A DS, Vader J, Zakeri R, Ravichandran A, AbouEzzadine O, Khazanie P, McNulty S. The tides of congestion during and after hospitalization for acute decompensated heart failure. J Cardiac Fail. 2013; 19(8):S81.

33. Grafft CA, McDonald FS, Ruud KL, Liesinger JT, Johnson MG, Naessens JM. Effect of hospital follow-up appointment on clinical event outcomes and mortality. Arch Intern Med. 2010;170(11): 955-960.

34. Lowrie R, Mair FS, Greenlaw N, et al; Heart Failure Optimal Outcomes from Pharmacy Study (HOOPS) Investigators. Pharmacist intervention in primary care to improve outcomes in patients with left ventricular systolic dysfunction. Eur Heart J. 2012;33(3):314-324.

35. Chaudhry SI, Mattera JA, Curtis JP, et al. Telemonitoring in patients with heart failure. N Engl J Med. 2010;363(24):2301-2309.

36. Koehler F, Winkler S, Schieber M, et al; Telemedical Interventional Monitoring in Heart Failure Investigators. Impact of remote telemedical management on mortality and hospitalizations in ambulatory patients with chronic heart failure: the telemedical interventional monitoring in heart failure study. Circulation. 2011;123(17):1873-1880.

37. Ong MK, Romano PS, Edgington S, et al. Effectiveness of remote patient monitoring after discharge of hospitalized patients with heart failure: the better effectiveness after transition - heart failure (BEAT-HF) randomized clinical trial. JAMA Intern Med. 2016;176(3):310-318.

38. Adamson PB. Pathophysiology of the transition from chronic compensated and acute decompensated heart failure: new insights from continuous monitoring devices. Curr Heart Fail Rep. 2009;6(4):287-292.
39. Adamson PB, Abraham WT, Aaron M, et al. CHAMPION trial rationale and design: the long-term safety and clinical efficacy of a wireless pulmonary artery pressure monitoring system. J Card Fail. 2011;17(1): $3-10$.

40. Abraham WT, Adamson PB, Bourge RC, et al; CHAMPION Trial Study Group. Wireless pulmonary artery haemodynamic monitoring in chronic heart failure: a randomised controlled trial. Lancet. 2011; 377(9766):658-666.

41. Abraham WT, Stevenson LW, Bourge RC, Lindenfeld JA, Bauman JG, Adamson PB; CHAMPION Trial Study Group. Sustained efficacy of pulmonary artery pressure to guide adjustment of chronic heart failure therapy: complete follow-up results from the CHAMPION randomised trial. Lancet. 2016;387(10017):453-461.

42. Mancini D, Colombo PC. Left ventricular assist devices: a rapidly evolving alternative to transplant. JAm Coll Cardiol. 2015;65(23):2542-2555.

43. Miller LW, Pagani FD, Russell SD, et al; HeartMate II Clinical Investigators. Use of a continuous-flow device in patients awaiting heart transplantation. N Engl J Med. 2007;357(9):885-896.

44. Slaughter MS, Rogers JG, Milano CA, et al; HeartMate II Investigators. Advanced heart failure treated with continuous-flow left ventricular assist device. $N$ Engl J Med. 2009;361(23):2241-2251.

45. Aaronson KD, Slaughter MS, Miller LW, et al; HeartWare Ventricular Assist Device (HVAD) Bridge to Transplant ADVANCE Trial Investigators. Use of an intrapericardial, continuous-flow, centrifugal pump in patients awaiting heart transplantation. Circulation. 2012;125(25):3191-3200.

46. Stewart GC, Givertz MM. Mechanical circulatory support for advanced heart failure: patients and technology in evolution. Circulation. 2012; 125(10):1304-1315.

47. Moazami N, Fukamachi K, Kobayashi M, et al. Axial and centrifugal continuous-flow rotary pumps: a translation from pump mechanics to clinical practice. $J$ Heart Lung Transplant. 2013;32(1):1-11.

48. Larose JA, Tamez D, Ashenuga M, Reyes C. Design concepts and principle of operation of the HeartWare ventricular assist system. ASAIOJ. 2010;56(4):285-289.

49. Mcllvennan CK, Magid KH, AmbardekarAV,Thompson JS, Matlock DD, Allen LA. Clinical outcomes after continuous-flow left ventricular assist device: a systematic review. Circ Heart Fail. 2014;7(6):1003-1013.

50. Heatley G, Sood P, Goldstein D, et al. Clinical trial design and rationale of the multicenter study of MagLev technology in patients undergoing mechanical circulatory support therapy with heartMate 3 (MOMENTUM 3) investigational device exemption clinical study protocol. $J$ Heart Lung Transplant. 2016;35(4):528-536.

51. Schmitto JD, Hanke JS, Rojas SV, Avsar M, Haverich A. First implantation in man of a new magnetically levitated left ventricular assist device (HeartMate III). J Heart Lung Transplant. 2015;34(6):858-860.

52. Netuka I, Sood P, Pya Y, et al. Fully magnetically levitated left ventricular assist system for treating advanced HF: a multicenter study. $J$ Am Coll Cardiol. 2015;66(23):2579-2589.

53. Zimpfer D, Netuka I, Schmitto JD, et al. Multicentre clinical trial experience with the HeartMate 3 left ventricular assist device: 30-day outcomes. Eur J Cardiothorac Surg. 2016;50(3):548-554.

54. Slaughter MS, Pagani FD, McGee EC, et al; HeartWare Bridge to Transplant ADVANCE Trial Investigators. HeartWare ventricular assist system for bridge to transplant: combined results of the bridge to transplant and continued access protocol trial. J Heart Lung Transplant. 2013;32(7):675-683.

55. Mehra MR, Naka Y, Uriel N, et al; MOMENTUM 3 Investigators. A fully magnetically levitated circulatory pump for advanced heart failure. N Engl J Med. Epub 2016 Nov 26.

56. Metra M. Tachycardia after a heart failure hospitalization: another piece of the puzzle? JACC Heart Fail. 2013;1(6):497-499.

57. Crozier I, Smith W. Modern device technologies. Heart Lung Circulation. 2012;21(6-7):320-327.

58. Shavelle D, Jermyn R. The CardioMEMS heart failure sensor: a procedural guide for implanting physicians. J Invasive Cardiol. 2016;28(7): 273-279. 
Vascular Health and Risk Management is an international, peerreviewed journal of therapeutics and risk management, focusing on concise rapid reporting of clinical studies on the processes involved in the maintenance of vascular health; the monitoring, prevention and treatment of vascular disease and its sequelae; and the involvement of metabolic disorders, particularly diabetes. This journal is indexed on PubMed Central and MedLine. The manuscript management system is completely online and includes a very quick and fair peer-review system, which is all easy to use. Visit http://www.dovepress.com/ testimonials.php to read real quotes from published authors. 